\title{
Simulation study of artifacts in Computed Tomography caused by metal implants
}

Gregor Imboden ${ }^{1}$, gregor.imboden@ @students.fhnw.ch

Prof. Dr. Erik Schkommodau', erik.schkommodau@fhnw.ch

${ }^{1}$ Institute for Medical- and Analytical technologies, University of Applied Sciences Northwestern Switzerland, Muttenz, Switzerland

\section{Introduction}

Artifacts caused by metal implants are a major hindrance for the observation of the bone implant interface in CT images. For the diagnosis of the implant loosening and the observation of osteogenic processes at the interface the reduction of imaging artifacts is necessary. We investigate the mechanism of appearance and constructive methods for the reduction of artifacts with the help of computer simulations and practical studies of implants created by additive manufacturing.

\section{Methods}

To simulate the process of 2D image reconstruction from 1D projections we intersect an analytically defined scene with pencil beams according to the parallel-beam geometry. The 2D image is reconstructed from the projections via the filtered backprojection (FBP) [1,2,3]. The projection acquisition model includes polychromaticity, noise and scatter. The reconstructions of different implant morphologies are then compared to the reference raster images based on regions of interest at the bone implant interface.

\section{Results}

The reconstructed images from the simulation and a real CT scanner were compared based on the point spread function (PSF) of a thin metal wire. The similarity of the PSF for both systems confirms the validity of the simulations. The artifacts induced by different implant morphologies were studied.

\section{Conclusion}

We developed a CT simulator for 2D image reconstruction from 1D projections and confirmed its validity to predict artifacts caused by metal implants. We used the simulator to study the artifacts induced by different implant morphologies.

[1] J. Hsieh, Computed Tomography: Principles, Design, Artifacts, and Recent Advances. SPIE Publications, 2003. [2] W. A. Kalender, Computed Tomography: Fundamentals, System Technology, Image Quality, Applications. John Wiley \& Sons, 2011.

[3] T. M. Buzug, Computed tomography: from photon statistics to modern cone-beam CT. Springer, 2008. 